\title{
Effects of Pioglitazone or Exercise in Older Adults with Mild Cognitive Impairment and Insulin Resistance: A Pilot Study
}

\author{
Kerry L. Hildreth Rachael E. Van Pelt Kerrie L. Moreau Jim Grigsby \\ Karin F. Hoth Victoria Pelak C. Alan Anderson Bennett Parnes \\ John Kittelson Pamela Wolfe Tammie Nakamura Sunny A. Linnebur \\ Jennifer M. Trujillo Christina L. Aquilante Robert S. Schwartz \\ Division of Geriatric Medicine, Department of Medicine, University of Colorado School of \\ Medicine, Aurora, Colo., USA
}

Key Words
Mild cognitive impairment $\cdot$ Insulin resistance $\cdot$ Pioglitazone $\cdot$ Exercise

\section{Abstract}

Aims: To examine the effects of pioglitazone or endurance exercise training on cognitive function in older adults with mild cognitive impairment (MCI) and insulin resistance. Methods: Seventy-eight adults (mean age \pm SD: $65 \pm 7$ years) with central obesity and MCI were randomized to 6 months of endurance exercise, pioglitazone or control. Results: Sixty-six participants completed the study. Exercise training did not significantly increase peak oxygen uptake compared to control $(p=0.12)$. Compared to control, insulin resistance improved in the pioglitazone group $(p=0.002)$ but not in the exercise group $(p=0.25)$. There was no measureable effect of pioglitazone or exercise on cognitive performance compared to control. Conclusion: In this pilot study, pioglitazone improved insulin resistance but not cognitive performance in older adults with $\mathrm{MCI}$ and insulin resistance.

(C) 2015 S. Karger AG, Basel

\section{Introduction}

Mild cognitive impairment (MCI) is defined as subjective and objective cognitive impairment without functional impairment [1]. Though a controversial entity, MCI - particularly the amnestic subtype - is thought in most cases to represent prodromal Alzheimer's disease (AD). The annual conversion rate from MCI to AD appears to range from 5 to $10 \%$ [2, 
3], depending on the population studied and the duration of follow-up, and is substantially higher than the annual incidence rate for dementia in the overall elderly population, estimated at 1-3\% [4-6]. Because MCI is often the earliest clinically detectable stage of AD, these patients may be more responsive to interventions to delay or prevent further cognitive decline than patients in later stages of disease.

Studies have repeatedly demonstrated that type 2 diabetes increases the risk of cognitive impairment and dementia, including AD [7-15]. Insulin resistance (IR), defined as a reduction in the effectiveness of insulin to regulate glucose utilization, is strongly associated with central obesity and has been linked to cognitive decline and AD even in the absence of type 2 diabetes $[16,17]$. Insulin is present in the brain, where it helps to support normal cognitive function [18], and abnormalities in insulin concentrations and activity have been observed in the brains of individuals with AD [19]. Interventions that target IR may therefore be helpful in delaying or preventing further cognitive decline in individuals with prodromal AD.

Both endurance exercise training (EET) [20] and thiazolidinediones (TZDs) [21] are effective in improving IR. Previous studies suggest EET [22] and TZDs [23-26] may each improve some aspects of cognitive function in individuals with MCI and/or early AD; however, no other studies have rigorously examined IR as a potential mechanism. The purpose of this double-blind, randomized, placebo-controlled pilot study was to determine whether improving IR with 6 months of EET or the TZD pioglitazone (PIO) would attenuate cognitive decline in older adults with both MCI and IR at baseline. We hypothesized that treatment with PIO or EET would have a beneficial effect on cognitive function, i.e. improvement or lesser decline relative to control (CON; placebo, no exercise), and that changes in cognitive performance would be correlated with changes in IR.

\section{Methods}

\section{Subjects}

Participants were sedentary, community-dwelling men and postmenopausal women 55 years of age or older. Participants met the diagnostic criteria for MCI [27] and Adult Treatment Panel (ATP) III criteria [28] for central obesity (waist circumference of $>88 \mathrm{~cm}$ for women and $>102 \mathrm{~cm}$ for men) as a proxy for IR. Participants were excluded if they had diabetes, dementia, neurologic comorbidities, exercise-limiting conditions, untreated depression, thyroid disease or vitamin $B_{12}$ deficiency, uncontrolled hypertension ( $>160 / 100 \mathrm{~mm} \mathrm{Hg}$ ), heart failure ( $\geq$ NYHA class II), contraindications to PIO or were taking cholinergic or anticholinergic medications (see online supplement for detailed exclusion criteria; for all online suppl. material, see www.karger.com/doi/10.1159/000371509).

\section{Study Design}

The study was approved by the Colorado Multiple Institutional Review Board, and all participants provided written informed consent. The study was designed, conducted and analyzed by the study team, with industry support limited to provision of PIO and matching placebo (Takeda Pharmaceuticals USA, Inc.). The study was a 6-month, randomized, controlled pilot study of blinded PIO or EET versus blinded CON. It is registered under Clinicaltrials.gov (NCT00736996). Randomization was performed using a documented permuted block randomization with random block sizes stratified by MCI subtype [amnestic (aMCI) or non-aMCI] and number of apolipoprotein E (APOE) $\varepsilon 4$ alleles $(0,1$ or 2$)$. Participants and investigators (except the study pharmacists and statistician) were blinded to drug treatment. Key outcome measures were assessed at baseline and 6 months by research personnel blinded to the treatment groups. 


\section{Screening}

Participants were screened for possible dementia or untreated depression. Those with evidence of dementia [Mini-Mental State Examination (MMSE) [29] score $<24$ or Clinical Dementia Rating (CDR) [30] sum of boxes score $>0.5$ ], functional impairment [Alzheimer's Disease Cooperative Study Activities of Daily Living (ADCS-ADL) basic inventory [31] <22], or untreated depression [Center for Epidemiologic Studies Depression (CES-D) [32] score $>36]$ were excluded.

\section{Determination of MCI}

MCI was determined by a multistep screening process using the revised Mayo Clinic criteria [27]. Participants were administered a neuropsychological testing battery consisting of assessments in 4 cognitive domains: memory (Visual Reproduction II [33], Logical Memory II [33], Rey Auditory Verbal Learning Test [34]), language (Boston Naming Test Naming Test [35], Category Fluency [36]), visuospatial (Block Design [37], Picture Completion [37]) and executive function (Trail Making Test B [38], Digit Symbol Test [37]). Raw test scores for these primary cognitive domain measures were transformed into age-adjusted scaled scores with a mean of 10 and a standard deviation (SD) of 3 using Mayo's Older American Normative Studies data [39-44]. Cognitive domain scores were calculated as the arithmetic mean of the normatively derived scaled scores for all of the tests in that domain. Thus, a domain score of 10 indicates exactly average performance, while a score of 13 indicates performance 1 SD above the mean relative to healthy age-matched peers. The cognitive domains, specific tests within each domain and scoring method were identical to that used in the Mayo Clinic Study of Aging [45, 46]. Participants who scored approximately 1 SD or more below the age-adjusted norms in any cognitive domain were considered for possible MCI and underwent a complete neurobehavioral examination by the study neurologist to rule out any identifiable neurologic disorder. Because aMCI and non-aMCI may represent different underlying pathologic processes and respond differently to the interventions, participants were further classified as having either aMCI (memory domain impaired) or non-aMCI (memory domain unimpaired). The determination of presence and type of MCI was made prior to randomization by consensus of the study investigators, neuropsychologists and neurologists after review of all available data and according to published criteria [27].

Exercise Testing

A standard treadmill stress test (modified Balke protocol) with electrocardiogram (ECG) and blood pressure monitoring was used to exclude participants with evidence of heart disease or other abnormal responses to exercise. Briefly, after a 5-min warm-up, walking speed was adjusted to elicit $70 \%$ of age-predicted maximal heart rate (HR max), and the treadmill grade increased 2\% every 2 min until volitional exhaustion. During the treadmill test exercise, peak oxygen consumption $\left(\mathrm{VO}_{2}\right.$ peak) and $\mathrm{HR}$ max were determined by open circuit spirometry (TrueMax 2400; Parvo-Medics, Sandy, Utah, USA) and ECG, respectively.

Drug Intervention

Participants randomized to the PIO or CON groups were given $30 \mathrm{mg}$ of PIO or matching placebo, respectively, to be taken once daily. After 1 month, the dose was increased to $45 \mathrm{mg}$ of PIO or matching placebo daily as tolerated. Adverse effects and clinical signs of heart failure were assessed at 2 weeks, and at monthly follow-up visits. Brain natriuretic peptide, hematocrit, alanine aminotransferase and fasting glucose were monitored throughout follow-up. If participants complained of adverse events that were intolerable, the dose of PIO or matching placebo was titrated down to maintain therapy. 
Hildreth et al.: Effects of Pioglitazone or Exercise in Older Adults with Mild Cognitive Impairment and Insulin Resistance: A Pilot Study

\section{Exercise Intervention}

Participants randomized to the EET group began by walking on the treadmill for 15-20 min at moderate intensity (50-60\% of $\mathrm{HR}$ max) as determined by baseline $\mathrm{VO}_{2}$ peak testing. Thereafter, exercise intensity was progressively increased to $80-85 \%$ of HR max, verified by heart rate monitors. Participants were asked to attend supervised 60-min exercise sessions 3 days per week at our Exercise Research Laboratory. The EET group did not receive placebo. There was no control exercise; rather, the CON group was instructed to maintain their current level of physical activity.

\section{Genetic Screening}

Participants were prospectively screened and stratified according to the number of copies of the $A P O E \varepsilon 4$ allele (0, 1 or 2). Briefly, genomic DNA was isolated from whole blood using a QIAamp DNA Mini Kit (Qiagen, Valencia, Calif., USA). The APOE rs429358 polymorphism (c.388 T>C; p.Cys130Arg) was genotyped in duplicate by PCR-pyrosequencing analysis (Qiagen) using the following primers: PCR forward, 5'-biotin-GCGGACATGGAGGACGTG-3'; PCR reverse, 5'-ACTGCCAGGCGCTTCTGC-3' , and sequencing, 5'-ACTGCACCAGGCGGC-3'

\section{Outcome Measures}

Cognitive Function

The 4 primary cognitive outcome variables included the change from baseline in the total score for each of the 4 cognitive domains (memory, language, visuospatial and executive function).

Secondary cognitive outcomes included change from baseline in the Alzheimer's Disease Cooperative Study (ADCS) MCI revision of the Alzheimer's Disease Assessment Scale-Cognitive subscale (ADAS-Cog) [47], Stroop Color-Word Interference test [48], the Digits Backward component of the Wechsler Adult Intelligence Scale-Revised Digit Span test [49], and the Clock Drawing test [50]. All cognitive testing was performed by a trained psychometrician in a private setting at baseline and 6 months.

\section{Insulin Sensitivity}

Single-stage $\left(40 \mathrm{mU} / \mathrm{m}^{2} / \mathrm{min}\right)$, 3-hour hyperinsulinemic, euglycemic clamps were performed at baseline and 6 months using the methods of DeFronzo et al. [51] and as previously described [52]. Clamps were performed at the University of Colorado Clinical and Translational Research Center following a 3-day standardized diet, 12-hour fast and $\geq 36 \mathrm{~h}$ after any vigorous exercise. After baseline measurement, insulin was infused (10 min prime, then constant rate) to maintain hyperinsulinemia. Arterialized [53] plasma glucose was measured at bedside every $5 \mathrm{~min}$, and 20\% dextrose infusion was variably adjusted to maintain euglycemia at $90 \mathrm{mg} / \mathrm{dl}$. Blood samples were collected at baseline and every $10 \mathrm{~min}$, under steadystate conditions, at the end of the insulin clamp (150, 160, 170 and $180 \mathrm{~min})$ for determination of plasma substrate and hormone concentrations. Whole-body glucose disposal rates (GDR; $\mathrm{mg} / \mathrm{kg} / \mathrm{min}$ ) were estimated from the steady-state glucose infusion rates.

\section{Body Composition}

Whole-body and regional (trunk, arm and leg) fat mass, lean mass, and bone mineral density were measured by dual X-ray absorptiometry (Hologic Delphi-W; software version 11.2 ) at baseline and 6 months.

Metabolic and Inflammatory Markers

Assays were performed after an overnight fast at baseline and 6 months. Serum cytokine levels included high-sensitivity C-reactive protein (immunoturbidimetric method; Beckman 
Hildreth et al.: Effects of Pioglitazone or Exercise in Older Adults with Mild Cognitive Impairment and Insulin Resistance: A Pilot Study

Coulter, Brea, Calif., USA), interleukin (IL)-6 and tumor necrosis factor (TNF)- $\alpha$ (ELISA; R\&D Systems, Minneapolis, Minn., USA). Hemoglobin ( $\mathrm{Hb}$ ) A1c was measured using HPLC, and lipids (total, high-density lipoprotein-C direct, low-density lipoprotein-C direct and triglycerides) were measured using enzymatic methods. All assays were performed by the University of Colorado Clinical and Translational Research Center Core Laboratory.

\section{Statistical Analysis}

The primary statistical analysis was an intention-to-treat analysis of the change from baseline in each of the 4 cognitive domains. The mean change with PIO or EET, separately, was compared with the mean improvement with $\mathrm{CON}$ in a linear regression conditioned on the stratification categories (MCI subtype and number of APOE $\varepsilon 4$ alleles) and the baseline level of the outcome variable. We defined statistical significance as a two-sided $\mathrm{p}$ value $<0.0125(=0.05 / 4)$ to adjust for multiple comparisons. Secondary analyses of the effects of treatment on IR (GDR) and $\mathrm{VO}_{2}$ peak were conducted using the same approach used for the primary analyses. Spearman's rank correlations were estimated to evaluate the association between change in GDR and the cognitive domain scores. Baseline characteristics of the treatment groups were compared using the global $\mathrm{F}$ test from a one-way ANOVA for continuous measures or a $\chi^{2}$ test for independent proportions for categorical measures. Power calculations suggested that a sample of 27 participants per group would provide at least $80 \%$ power to detect a change of approximately $0.9 \mathrm{SD}$ across each of the 4 cognitive domains. All analyses were prespecified and prioritized in a statistical analysis plan filed prior to data analysis. All analyses were conducted using SAS version 9.2 (SAS Institute Inc., Cary, N.C., USA).

\section{Results}

\section{Participant Characteristics}

Seventy-eight eligible volunteers were randomized to intervention; of these, 12 (15\%) dropped out and refused follow-up (fig. 1). The 66 participants who completed the intervention (85\%) were included in the analysis. Participants who dropped out were younger and more likely to be in the EET group, but were not different from completers in any other baseline characteristics or primary measures of cognitive function. Among the participants included in the analysis, baseline differences among the 3 groups were found in ethnicity and scores on the CES-D, ADAS-Cog and Digits Backward (table 1). Ninety percent of the sample was characterized as having aMCI.

\section{Intervention Compliance, Physiological Changes and Adverse Effects}

Adherence to the study drug determined by pill count at monthly visits was $76 \%$ in the PIO group and $89 \%$ in the CON group ( $p=0.22$ ). The average PIO dose at the end of the study was $39 \mathrm{mg} ; 85 \%$ of PIO participants were titrated to and remained at the $45-\mathrm{mg}$ dose. Adherence to exercise (attending $\geq 65 \%$ of sessions) was $82 \%$ in the EET group. On average, the EET group exercised $2.6 \pm 1.0$ days per week and $97.9 \pm 52.6$ min per week. The average HR during exercise was $80 \pm 5 \%$ of HR max. The interventions were well tolerated. Expected increases in upper respiratory infections (6 PIO; 0 CON) and peripheral edema (4 PIO; 2 CON) were seen in the PIO group. There were no cases of new or worsening heart failure in the PIO group. Musculoskeletal complaints were more common in the EET group (5 EET; 0 CON).

Oxygen uptake at peak exercise $\left(\mathrm{VO}_{2}\right.$ peak, $\left.\mathrm{ml} / \mathrm{kg} / \mathrm{min}\right)$ increased by $12.4 \%$ in the EET group compared with 6.0 and $2.0 \%$ in the CON and PIO groups, respectively; however, none of these changes was statistically significant (table 2). Compared to CON, GDR increased and fasting insulin decreased in the PIO group, but not in the EET group. There was no change in 


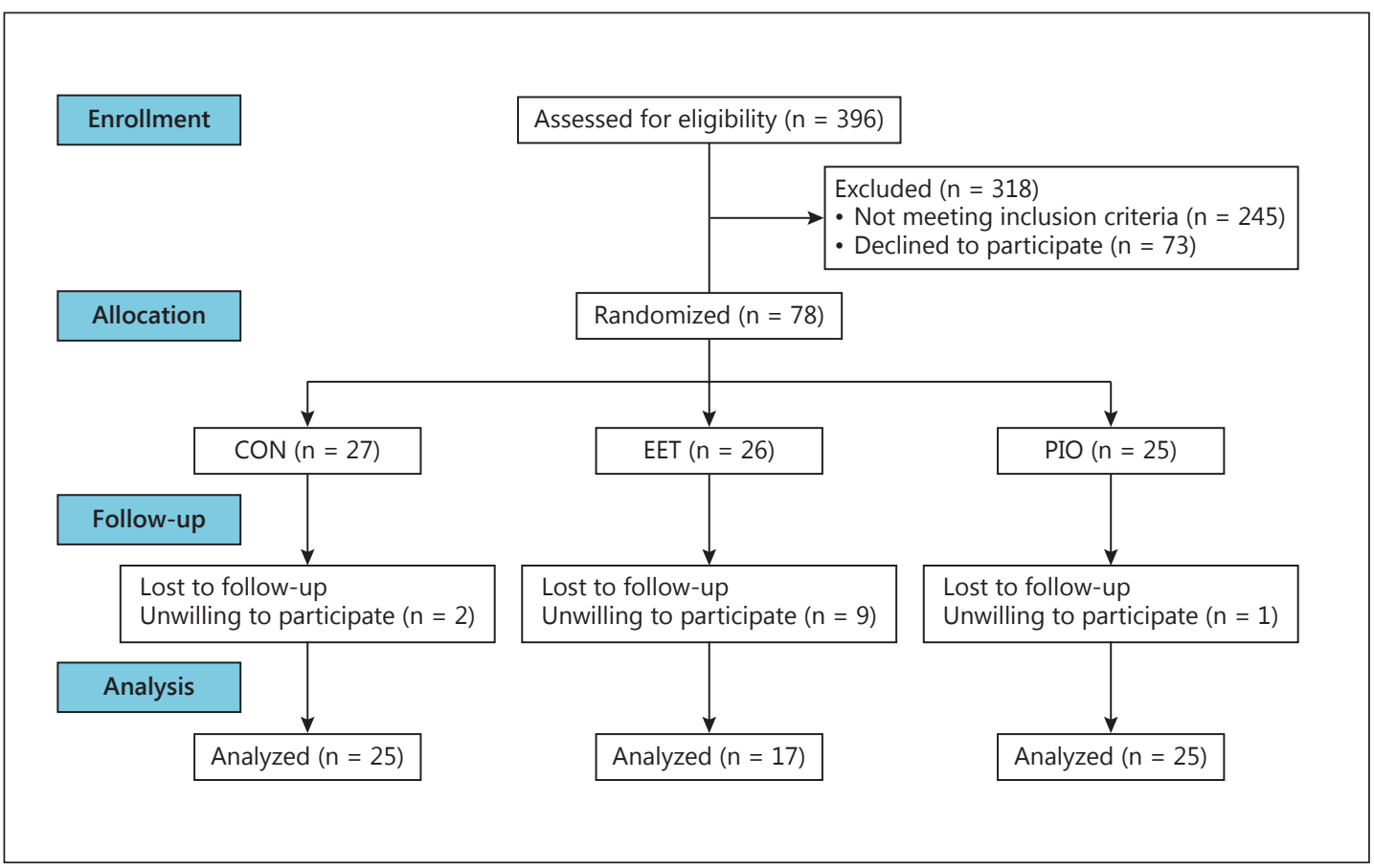

Fig. 1. Participant flow diagram.

HbA1c in the PIO or EET group compared with CON. Fasting triglycerides and IL-6 decreased in all groups; however, neither intervention group differed from CON. Fat mass decreased in the EET group compared to CON; there were no other significant changes from baseline to 6 months in body composition, blood pressure, metabolic or inflammatory markers.

\section{Cognitive Change Analyses}

Cognitive performance did not change significantly from baseline to 6 months in any group in any of the 4 domains (table 3). There was no measureable effect of either EET or PIO on the change in any cognitive domain score compared with CON. The only statistically significant difference in the secondary cognitive outcomes was greater improvement on the ADAS-Cog in EET $[-1.6,95 \%$ confidence interval (CI) $-4.9,1.6]$ compared with CON $(-0.3$, 95\% CI $-3.5,3.0 ; \mathrm{p}=0.05$ ).

Exploratory analysis of the individual cognitive tests comprising the various domains showed that scores on the Block Design test improved in the EET group, although the change was not significantly different from CON; there was no change in the PIO group. Performance on the Visual Reproduction test significantly declined in the participants in the PIO group compared to CON. There were no significant correlations in any group between either change in GDR or change in $\mathrm{VO}_{2}$ peak and cognitive performance.

\section{Discussion}

In older adults with MCI and IR, 6 months of PIO had no apparent effect on cognitive performance relative to CON. Six months of EET also had no apparent effect on cognitive performance relative to $\mathrm{CON}$, although the high attrition and lack of training effect in the EET group limits the interpretation of these results. To our knowledge, this pilot study is the first 
Hildreth et al.: Effects of Pioglitazone or Exercise in Older Adults with Mild Cognitive Impairment and Insulin Resistance: A Pilot Study

Table 1. Demographic and clinical characteristics of the participants at baseline

\begin{tabular}{|c|c|c|c|c|}
\hline & $\begin{array}{l}\mathrm{CON} \\
(\mathrm{n}=25)\end{array}$ & $\begin{array}{l}\text { EET } \\
(n=17)\end{array}$ & $\begin{array}{l}\text { PIO } \\
(n=24)\end{array}$ & $\mathrm{p}$ value \\
\hline Age, years & $64 \pm 7$ & $65 \pm 6$ & $68 \pm 7$ & 0.09 \\
\hline Females & $15(60.0)$ & 7 (41.2) & $13(54.2)$ & 0.48 \\
\hline White & $23(92.0)$ & $15(88.2)$ & $20(83.3)$ & 0.51 \\
\hline Hispanic & $0(0.0)$ & $0(0.0)$ & $4(16.7)$ & 0.03 \\
\hline Education, years & $16 \pm 2$ & $16 \pm 3$ & $16 \pm 2$ & 0.62 \\
\hline aMCI & $22(88.0)$ & $15(88.2)$ & $22(91.7)$ & 0.90 \\
\hline APOE $\varepsilon 4$ alleles & & & & 0.97 \\
\hline 0 & $18(72.0)$ & $11(64.7)$ & $18(75.0)$ & \\
\hline 1 & $6(24.0)$ & $5(29.4)$ & $5(20.8)$ & \\
\hline 2 & $1(4.0)$ & $1(5.9)$ & $1(4.2)$ & \\
\hline Antihypertensive medication & $10(40.0)$ & $8(47.1)$ & $14(58.3)$ & 0.43 \\
\hline Lipid-lowering medication & $8(32.0)$ & $7(41.2)$ & $8(33.3)$ & 0.82 \\
\hline Antidepressant medication & $4(16.0)$ & $4(23.5)$ & 7 (29.2) & 0.54 \\
\hline CES-D & $11.1 \pm 7.9$ & $9.1 \pm 6.1$ & $6.6 \pm 3.7$ & 0.04 \\
\hline MMSE & $28.8 \pm 1.3$ & $28.6 \pm 1.1$ & $28.4 \pm 1.3$ & 0.64 \\
\hline CDR sum of boxes & $0.2 \pm 0.3$ & $0.1 \pm 0.2$ & $0.2 \pm 0.2$ & 0.29 \\
\hline \multicolumn{5}{|c|}{ Body composition, metabolic and inflammatory measures } \\
\hline Weight, kg & $84.1 \pm 14.2$ & $85.3 \pm 17.5$ & $84.0 \pm 14.8$ & 0.96 \\
\hline BMI & $29 \pm 4$ & $29 \pm 3$ & $31 \pm 5$ & 0.40 \\
\hline Waist circumference, $\mathrm{cm}$ & $104.0 \pm 10.3$ & $104.1 \pm 12.1$ & $106.3 \pm 9.3$ & 0.70 \\
\hline Fat mass, kg & $32.1 \pm 7.4$ & $30.4 \pm 7.4$ & $32.5 \pm 9.8$ & 0.72 \\
\hline Fat-free mass, kg & $52.0 \pm 11.5$ & $54.9 \pm 3.3$ & $51.5 \pm 11.7$ & 0.65 \\
\hline $\mathrm{SBP}, \mathrm{mm} \mathrm{Hg}$ & $121 \pm 13$ & $125 \pm 13$ & $127 \pm 11$ & 0.19 \\
\hline DBP, mm Hg & $76 \pm 9$ & $76 \pm 10$ & $77 \pm 7$ & 0.87 \\
\hline $\mathrm{VO}_{2}$ peak, $\mathrm{l} / \mathrm{min}$ & $2.0 \pm .5$ & $1.9 \pm 0.5$ & $1.7 \pm 0.5$ & 0.22 \\
\hline Glucose disposal rate, $\mathrm{mg} / \mathrm{kg} / \mathrm{min}$ & $5.7 \pm 2.0$ & $5.6 \pm 2.2$ & $5.9 \pm 2.6$ & 0.92 \\
\hline $\mathrm{HbA} 1 \mathrm{c}, \%$ & $5.9 \pm 0.4$ & $6.0 \pm 0.3$ & $5.9 \pm 0.5$ & 0.67 \\
\hline Fasting glucose, $\mathrm{mg} / \mathrm{dl}$ & $101 \pm 12$ & $102 \pm 9$ & $101 \pm 13$ & 0.98 \\
\hline Fasting insulin, $\mu \mathrm{U} / \mathrm{ml}$ & $16.8 \pm 7.8$ & $17.1 \pm 8.9$ & $14.5 \pm 6.2$ & 0.45 \\
\hline Leptin, ng/ml & $22.2 \pm 13.8$ & $18.4 \pm 10.1$ & $27.3 \pm 22.7$ & 0.25 \\
\hline Total cholesterol, mg/dl & $171 \pm 36$ & $177 \pm 44$ & $162 \pm 36$ & 0.46 \\
\hline Triglycerides, mg/dl & $136 \pm 65$ & $200 \pm 226$ & $121 \pm 42$ & 0.12 \\
\hline HDL cholesterol, mg/dl & $41 \pm 10$ & $40 \pm 10$ & $40 \pm 8$ & 0.92 \\
\hline LDL cholesterol, mg/dl & $103 \pm 27$ & $103 \pm 37$ & $98 \pm 31$ & 0.84 \\
\hline $\mathrm{CRP}, \mathrm{mg} / \mathrm{l}$ & $2.9 \pm 2.7$ & $3.9 \pm 5.0$ & $3.0 \pm 3.6$ & 0.70 \\
\hline IL-6, pg/ml & $1.8 \pm 1.1$ & $10.4 \pm 32.7$ & $1.9 \pm 1.2$ & 0.21 \\
\hline TNF- $\alpha, \mathrm{pg} / \mathrm{ml}$ & $1.5 \pm 0.9$ & $1.4 \pm 0.5$ & $1.8 \pm 2.2$ & 0.71 \\
\hline \multicolumn{5}{|l|}{ Cognitive measures } \\
\hline Memory domain & $9.7 \pm 2.1$ & $9.5 \pm 2.5$ & $9.2 \pm 2.5$ & 0.80 \\
\hline Language domain & $10.8 \pm 2.3$ & $11.0 \pm 1.7$ & $10.0 \pm 1.9$ & 0.26 \\
\hline Visuospatial domain & $11.0 \pm 2.0$ & $11.6 \pm 2.3$ & $10.6 \pm 1.8$ & 0.29 \\
\hline Executive domain & $9.1 \pm 2.1$ & $10.8 \pm 2.3$ & $9.9 \pm 2.3$ & 0.07 \\
\hline ADAS-Cog & $5.4 \pm 2.8$ & $5.5 \pm 1.8$ & $7.2 \pm 2.8$ & 0.04 \\
\hline Stroop Color-Word response time, ms & $1,474 \pm 374$ & $1,339 \pm 346$ & $1,574 \pm 516$ & 0.22 \\
\hline Digits Backward & $6.3 \pm 1.7$ & $7.8 \pm 1.9$ & $6.3 \pm 1.8$ & 0.01 \\
\hline Clock Drawing & $19.3 \pm 0.9$ & $19.4 \pm 0.9$ & $19.3 \pm 1.0$ & 0.95 \\
\hline
\end{tabular}

Values are presented as means \pm SD or $\mathrm{n}(\%)$. BMI = Body mass index; SBP = systolic blood pressure; $\mathrm{DBP}=$ diastolic blood pressure; HDL = high-density lipoprotein; LDL = low-density lipoprotein; $\mathrm{CRP}=$ C-reactive protein. 
Hildreth et al.: Effects of Pioglitazone or Exercise in Older Adults with Mild Cognitive Impairment and Insulin Resistance: A Pilot Study

Table 2. Change from baseline in body composition and metabolic and inflammatory markers

\begin{tabular}{|c|c|c|c|c|c|}
\hline & \multirow[t]{2}{*}{ CON $(n=25)$} & \multirow[t]{2}{*}{$\operatorname{EET}(n=17)$} & \multirow[t]{2}{*}{ PIO $(n=24)$} & \multicolumn{2}{|l|}{$\mathrm{p}$ value } \\
\hline & & & & $\begin{array}{l}\text { EET vs. } \\
\text { CON }\end{array}$ & $\begin{array}{l}\text { PIO vs. } \\
\text { CON }\end{array}$ \\
\hline Weight, kg & $-2.5(-6.0,1.0)$ & $-3.6(-7.1,-0.1)$ & $-2.1(-5.6,1.4)$ & 0.17 & 0.59 \\
\hline Fat mass, kg & $-2.3(-5.2,0.6)$ & $-3.7(-6.6,-0.8)$ & $-1.8(-4.7,1.2)$ & 0.03 & 0.31 \\
\hline Fat-free mass, kg & $-0.3(-2.3,1.7)$ & $0.1(-2.0,2.1)$ & $-0.5(-2.5,1.6)$ & 0.47 & 0.62 \\
\hline BMI & $-0.9(-2.1,0.4)$ & $-1.2(-2.4,0.1)$ & $-0.6(-1.9,0.6)$ & 0.25 & 0.32 \\
\hline $\mathrm{SBP}, \mathrm{mm} \mathrm{Hg}$ & $-5.4(-20,9.4)$ & $-10(-25,4.5)$ & $-10(-25,4.4)$ & 0.13 & 0.11 \\
\hline DBP, mm Hg & $3.5(-5.4,12.5)$ & $0.0(-9.0,9.1)$ & $0.5(-8.5,9.6)$ & 0.08 & 0.10 \\
\hline $\mathrm{VO}_{2}$ peak, $1 / \mathrm{min}$ & $0.0(-0.4,0.5)$ & $0.2(-0.2,0.6)$ & $0.0(-0.4,0.5)$ & 0.11 & 0.99 \\
\hline $\mathrm{VO}_{2}$ peak, $\mathrm{ml} / \mathrm{kg} / \mathrm{min}$ & $1.3(-4.1,6.7)$ & $3.2(-2.3,8.7)$ & $0.9(-4.6,6.4)$ & 0.12 & 0.72 \\
\hline \multicolumn{6}{|l|}{ Metabolic markers } \\
\hline $\mathrm{HbA1c}, \%$ & $-0.1(-0.2,0.0)$ & $-0.0(-0.2,0.1)$ & $-0.2(-0.4,-0.1)$ & 0.65 & 0.20 \\
\hline Leptin, ng/ml & $-0.4(-4.2,3.4)$ & $-0.5(-5.0,4.1)$ & $0.5(-3.4,4.5)$ & 0.99 & 0.72 \\
\hline Fasting glucose, $\mathrm{mg} / \mathrm{dl}$ & $-1.4(-3.7,1.0)$ & $0.7(-2.1,3.5)$ & $-2.3(-4.7,0.1)$ & 0.27 & 0.60 \\
\hline Fasting insulin, $\mu \mathrm{U} / \mathrm{ml}$ & $-0.4(-3.2,2.5)$ & $3.3(-0.0,6.7)$ & $-6.7(-9.6,-3.8)$ & 0.10 & 0.003 \\
\hline Glucose disposal rate, $\mathrm{mg} / \mathrm{kg} / \mathrm{min}$ & $0.1(-0.6,0.8)$ & $0.7(-0.1,1.6)$ & $1.7(0.9,2.4)$ & 0.25 & 0.002 \\
\hline Total cholesterol, mg/dl & $-1.5(-10.0,6.9)$ & $-5.7(-15.3,3.8)$ & $-7.0(-15.9,1.9)$ & 0.52 & 0.39 \\
\hline Triglycerides, $\mathrm{mg} / \mathrm{dl}$ & $-21.9(-39.9,-3.9)$ & $-24.3(-44.8,-3.8)$ & $-28.5(-47.5,-9.4)$ & 0.86 & 0.62 \\
\hline HDL cholesterol, mg/dl & $1.7(-5.3,8.7)$ & $-1.0(-9.1,7.1)$ & $-5.1(-12.5,2.2)$ & 0.62 & 0.18 \\
\hline LDL cholesterol, mg/dl & $-0.5(-2.7,1.6)$ & $-2.0(-4.4,0.4)$ & $0.6(-1.6,2.9)$ & 0.38 & 0.46 \\
\hline \multicolumn{6}{|l|}{ Inflammatory markers } \\
\hline $\mathrm{CRP}, \mathrm{mg} / \mathrm{l}$ & $1.4(-2.2,4.9)$ & $-1.0(-5.3,3.2)$ & $2.9(-0.8,6.6)$ & 0.39 & 0.55 \\
\hline IL-6, pg/ml & $-1.5(-2.1,-1.0)$ & $-2.2(-2.8,-1.5)$ & $-1.8(-2.3,-1.2)$ & 0.15 & 0.57 \\
\hline TNF- $\alpha, p g / m l$ & $0.1(-0.2,0.4)$ & $-0.2(-0.6,0.2)$ & $-0.2(-0.5,0.1)$ & 0.22 & 0.20 \\
\hline
\end{tabular}

Values are unit change $(95 \% \mathrm{CI})$. Estimates were done in a regression model controlling for baseline value, number of $A P O E \varepsilon 4$ alleles, and MCI subtype.

to examine both of these interventions in a rigorous randomized controlled trial in a population at high risk for cognitive decline by virtue of having both MCI and IR.

TZDs are agonists of the peroxisome proliferator-activated receptor- $\gamma$ (PPAR- $\gamma$ ) that decrease plasma insulin and glucose, and improve insulin sensitivity [21]. TZDs also have ancillary properties that make them an attractive potential therapy for the prevention or delay of cognitive decline and AD. Specifically, TZDs have been shown to decrease markers of inflammation and promote vasodilation in humans [21]. Recent evidence suggests that PPAR- $\gamma$ agonists may also reduce accumulation and increase clearance of $\beta$-amyloid [54]. Two studies of rosiglitazone have found improvements in some areas of cognitive function in participants with aMCI and mild to moderate AD $[23,24]$. However, more recent studies failed to find any effect of rosiglitazone on cognition in participants with mild to moderate AD either as monotherapy [55] or as an adjunct to treatment with cholinesterase inhibitors [56]. To our knowledge, only 2 other groups have studied the effects of PIO on cognition in participants with MCI or AD. One group reported improvements in the ADAS-Cog and WMS-R logical memory in participants with mild to moderate $\mathrm{AD}$ or aMCI and noninsulin-dependent type 2 diabetes who received PIO for 6 months (15-30 mg daily) compared with placebo in a randomized open controlled study $[25,26]$. A second double-blind randomized controlled trial of the safety of PIO (up to $45 \mathrm{mg}$ daily) in nondiabetic participants with probable AD found that PIO was well tolerated over an 18-month period, but had no effect on the secondary cognitive outcomes [57].

To our knowledge, ours is the first study to rigorously investigate the mechanistic role of IR in cognitive decline. We found that PIO significantly improved IR but not cognitive function. Although GDR determined by hyperinsulinemic-euglycemic clamp is the gold standard for 
Table 3. Change in cognitive function scaled scores from baseline

\begin{tabular}{lccccc}
\hline & CON $(\mathrm{n}=25)$ & EET $(\mathrm{n}=17)$ & PIO $(\mathrm{n}=24)$ & p value \\
\cline { 3 - 6 } & & & & $\begin{array}{l}\text { EET vs. PIO vs. } \\
\text { CON }\end{array}$ & CON \\
\hline Memory Domain Scaled Score & & & & & \\
Visual Reproduction II [33] & $1.2(-1.0,3.4)$ & $0.7(-1.5,2.8)$ & $0.8(-1.4,2.9)$ & 0.29 & 0.35 \\
Logical Memory II [33] & $1.4(-2.4,5.1)$ & $0.2(-3.5,3.9)$ & $-0.5(-4.3,3.2)$ & 0.15 & 0.009 \\
Rey Auditory Verbal Learning Test [34] & $1.5(-2.5,5.5)$ & $0.9(-3.0,4.8)$ & $1.8(-2.1,5.8)$ & 0.48 & 0.73 \\
\hline Language Domain Scaled Score & $0.8(-2.3,4.0)$ & $1.0(-2.1,4.1)$ & $1.0(-2.1,4.2)$ & 0.82 & 0.74 \\
Boston Naming Test [35] & $0.4(-1.9,2.6)$ & $0.6(-1.6,2.8)$ & $0.4(-1.8,2.6)$ & 0.62 & 0.95 \\
Category Fluency [36] & $0.8(-2.7,4.4)$ & $0.5(-3.1,4.0)$ & $1.0(-2.5,4.5)$ & 0.61 & 0.85 \\
\hline Visuospatial Domain Scaled Score & $-0.4(-2.8,2.0)$ & $0.5(-1.9,2.8)$ & $-0.4(-2.8,2.0)$ & 0.10 & 0.97 \\
Block Design [37] & $0.8(-1.2,2.8)$ & $1.1(-0.9,3.1)$ & $0.3(-1.7,2.3)$ & 0.51 & 0.19 \\
Picture Completion [37] & $1.9(-0.9,4.7)$ & $2.8(0.1,5.5)$ & $1.5(-1.3,4.3)$ & 0.14 & 0.41 \\
\hline Executive Domain Scaled Score & $0.1(-2.7,2.9)$ & $-0.1(-2.9,2.6)$ & $-0.6(-3.4,2.1)$ & 0.73 & 0.20 \\
Trail Making Test B [38] & $0.9(-1.1,2.9)$ & $0.7(-1.2,2.6)$ & $1.0(-0.9,3.0)$ & 0.64 & 0.66 \\
Digit Symbol Test [37] & $1.5(-1.6,4.5)$ & $0.8(-2.1,3.7)$ & $1.4(-1.6,4.4)$ & 0.29 & 0.89 \\
\hline
\end{tabular}

Values are unit change $(95 \% \mathrm{CI})$.

assessing insulin sensitivity, there is no accepted cutoff value for determining IR; rather, GDR exists on a spectrum from extremely sensitive to extremely resistant. A GDR of $<4.7 \mathrm{mg} / \mathrm{kg} /$ min has been proposed as a conservative estimate for IR based on data from 18 independent $40 \mathrm{mU} / \mathrm{m}^{2} / \mathrm{min}$ clamp studies [58]. The mean GDR in the present study was $5.68 \pm 2.21 \mathrm{mg} /$ $\mathrm{kg} / \mathrm{min}$, suggesting the degree of IR was mild. Although we found no correlation between change in GDR and change in cognitive performance, a larger sample with a greater distribution of GDR values may be needed to detect an association.

The participants in this study differed from those in previous studies of TZDs in several potentially important ways. First, the degree of cognitive impairment was very mild, as evidenced by baseline MMSE, CDR and cognitive domain scores. Thus, compared to previous studies which have primarily included patients with AD or more advanced MCI, participants in the present study were far less impaired. Second, both aMCI and non-aMCI participants were included. These subtypes likely have different underlying pathology, with the former more strongly associated with $\mathrm{AD}$, and may therefore respond differently to the interventions. With the exception of a decrease in TNF- $\alpha$ in the EET group compared to CON, results from exploratory post hoc analyses on $90 \%$ of the sample with aMCI were not significantly different from those on the full sample (online suppl. table 1). Third, $29 \%$ of the participants in this study were $A P O E \& 4$ carriers, whereas previous studies of MCI patients have reported rates $>40 \%[24,26]$. In exploratory post hoc analyses, memory domain scores improved among noncarriers assigned to PIO, but improvements were not significantly different from CON (online suppl. table 2). Conversely, visuospatial domain scores decreased among noncarriers in the PIO group compared to CON. Among noncarriers assigned to EET, IL-6 levels decreased and fasting insulin increased compared to CON. These results must be interpreted cautiously due to the small sample sizes, but they suggest there may be differential responses to the interventions between $A P O E \varepsilon 4$ carriers and noncarriers. Participants were rigorously evaluated to rule out cognitive impairment due to vascular disease or other medical conditions, but it is possible that this population was skewed toward the subset of individuals with $\mathrm{MCI}$ who do not convert to AD, particularly as biomarkers of AD were not assessed. These characteristics may explain the absence of decline in any cognitive domain in the CON group; 
Hildreth et al.: Effects of Pioglitazone or Exercise in Older Adults with Mild Cognitive Impairment and Insulin Resistance: A Pilot Study

indeed, small improvements in all domains were observed across groups. The mild degree of impairment may have contributed to a learning effect and made it more difficult to detect changes in cognitive performance.

It is possible that actions of PIO other than reducing IR may be important for supporting cognitive function. Although IL-6 and triglycerides decreased in the PIO group, the changes were not different from CON. Markers of amyloid were not assessed in this study. Although IR has been repeatedly associated with an increased risk of AD and IR has been demonstrated in the brains of persons with $\mathrm{AD}$ [59], the relation between peripheral and central IR with respect to cognition is unclear, and improvements in peripheral IR may not translate into central improvements, which are not easily measured. This is supported by recent studies demonstrating cognitive benefits of intranasal insulin which bypasses the periphery and delivers insulin directly to the brain [60].

Data from epidemiologic [61] and randomized controlled trials [22, 62-64] strongly support a beneficial role for physical activity and exercise on cognitive function. The potential mechanisms by which exercise may improve cognition are not known, but may include improvements in IR [20]. In the present study, attrition in the EET group was high, and while session attendance was high, adherence to the prescribed exercise intervention was suboptimal as evidenced by the lack of improvement in $\mathrm{VO}_{2}$ peak and IR; thus, we cannot draw any conclusions about the effects of aerobic exercise in this study. Our group has successfully trained older adults with a similar intervention in previous studies [65-69], and the intensity of the exercise intervention in the present study was similar to that used by Baker et al. [64] in cognitively normal participants, but less intense than that in previous studies in MCI patients. It has been suggested that cognitive function may affect adherence to exercise [70] and that achieving a higher degree of exercise intensity may require protocol adaptations in a cognitively impaired population.

This pilot study has some important limitations. Sample sizes for each group were slightly smaller than planned for, and attrition in the EET group was higher than anticipated; thus, the study was underpowered. However, given that there was no significant change in cognitive domain scores in any group, the likelihood of a type II error is small. We did not include a true active control for the exercise group (e.g. stretching) or a combined PIO + EET group. It is possible that practice effects may underlie the small improvements seen from baseline to 6 months; however, use of change scores would likely have equalized these effects across groups. While we used a comprehensive cognitive testing battery, it is possible that other aspects of cognition were affected by the intervention. We did not measure any biomarkers of $\mathrm{AD}$ [1] and thus cannot be certain that all of the participants had early AD, although $90 \%$ had aMCI, which is generally thought to represent early AD. We used the ATP-III criteria for central obesity to coarsely screen for IR. Although waist circumference is a strong predictor of IR [71, 72], not all individuals with central obesity demonstrate IR. The average fasting glucose and HbA1c of the population were within the 'prediabetes' range [73], but the degree of IR was mild, with some participants below the lower range. It is possible that outcomes may differ in a more insulin-resistant population. Finally, the participants were primarily white, highly educated and generally healthy. Whether our findings would extend to other populations is not known.

\section{Conclusion}

Despite evidence supporting a mechanistic role of IR in the development and progression of cognitive impairment, 6 months of PIO did not appear to improve cognitive performance in older adults with MCI and IR in this pilot study. The effects of EET were inconclusive due 
to the high attrition rate and lack of training effect. The current lack of effective treatments for $\mathrm{AD}$ and the rapidly increasing burden of the disease highlight the urgent need for interventions to delay or prevent decline in individuals with prodromal AD. Studies of longer duration in patients with a range of levels of cognitive impairment along the spectrum to AD and exercise interventions tailored to those with cognitive impairment may be helpful in determining the effects of interventions targeting IR on cognitive function.

\section{Acknowledgements}

The authors thank Suzanne Craft, PhD, and Ronald Petersen, MD, PhD, for their contributions. This work was supported by the National Institutes of Health (NIH)/National Institute on Aging grant R01AG028746 and NIH/National Center for Advancing Translational Sciences Colorado Clinical and Translational Sciences Institute grant UL1 TR001082. Contents are the authors' sole responsibility and do not necessarily represent official NIH views. Pioglitazone and placebo tablets were donated by Takeda Pharmaceuticals USA Inc.

\section{Disclosure Statement}

The authors report no conflicts.

\section{References}

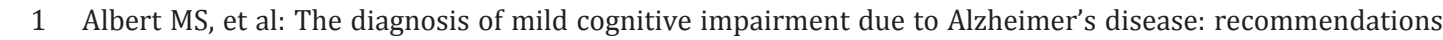
from the National Institute on Aging-Alzheimer's Association workgroups on diagnostic guidelines for Alzheimer's disease. Alzheimers Dement 2011;7:270-279.

2 Mitchell AJ, Shiri-Feshki M: Rate of progression of mild cognitive impairment to dementia - meta-analysis of 41 robust inception cohort studies. Acta Psychiatr Scand 2009;119:252-265.

-3 Mitchell AJ, Shiri-Feshki M: Temporal trends in the long term risk of progression of mild cognitive impairment: a pooled analysis. J Neurol Neurosurg Psychiatry 2008;79:1386-1391.

4 Boyle PA, et al: Mild cognitive impairment: risk of Alzheimer disease and rate of cognitive decline. Neurology 2006;67:441-445.

5 Petersen RC, et al: Mild cognitive impairment: clinical characterization and outcome. Arch Neurol 1999;56: 303-308.

-6 Larrieu S, et al: Incidence and outcome of mild cognitive impairment in a population-based prospective cohort. Neurology 2002;59:1594-1599.

7 Akomolafe A, et al: Diabetes mellitus and risk of developing Alzheimer disease: results from the Framingham Study. Arch Neurol 2006;63:1551-1555.

-8 Arvanitakis Z, et al: Diabetes mellitus and risk of Alzheimer disease and decline in cognitive function. Arch Neurol 2004;61:661-666.

-9 Leibson CL, et al: The risk of dementia among persons with diabetes mellitus: a population-based cohort study. Ann NY Acad Sci 1997;826:422-427.

10 Leibson CL, et al: Risk of dementia among persons with diabetes mellitus: a population-based cohort study. Am J Epidemiol 1997;145:301-308.

11 Luchsinger JA, et al: Diabetes mellitus and risk of Alzheimer's disease and dementia with stroke in a multiethnic cohort. Am J Epidemiol 2001;154:635-641.

$\checkmark 12$ MacKnight C, et al: Diabetes mellitus and the risk of dementia, Alzheimer's disease and vascular cognitive impairment in the Canadian Study of Health and Aging. Dement Geriatr Cogn Disord 2002;14:77-83.

13 Ott A, et al: Diabetes mellitus and the risk of dementia: The Rotterdam Study. Neurology 1999;53:1937-1942.

14 Peila R, Rodriguez BL, Launer LJ: Type 2 diabetes, APOE gene, and the risk for dementia and related pathologies: The Honolulu-Asia Aging Study. Diabetes 2002;51:1256-1262.

15 Xu WL, et al: Diabetes mellitus and risk of dementia in the Kungsholmen project: a 6-year follow-up study. Neurology 2004;63:1181-1186.

16 Luchsinger JA, et al: Hyperinsulinemia and risk of Alzheimer disease. Neurology 2004;63:1187-1192. 
Hildreth et al.: Effects of Pioglitazone or Exercise in Older Adults with Mild Cognitive Impairment and Insulin Resistance: A Pilot Study

Kuusisto J, et al: Association between features of the insulin resistance syndrome and Alzheimer's disease independently of apolipoprotein E4 phenotype: cross sectional population based study. BMJ 1997;315:10451049.

18 Craft S: The role of metabolic disorders in Alzheimer disease and vascular dementia: two roads converged Arch Neurol 2009;66:300-305.

19 Steen E, et al: Impaired insulin and insulin-like growth factor expression and signaling mechanisms in Alzheimer's disease - is this type 3 diabetes? J Alzheimers Dis 2005;7:63-80.

20 Ryan AS: Insulin resistance with aging: effects of diet and exercise. Sports Med 2000;30:327-346.

21 Yki-Jarvinen H: Thiazolidinediones. N Engl J Med 2004;351:1106-1118.

-22 Heyn P, Abreu BC, Ottenbacher KJ: The effects of exercise training on elderly persons with cognitive impairment and dementia: a meta-analysis. Arch Phys Med Rehabil 2004;85:1694-1704.

23 Watson GS, et al: Preserved cognition in patients with early Alzheimer disease and amnestic mild cognitive impairment during treatment with rosiglitazone: a preliminary study. Am J Geriatr Psychiatry 2005;13:950958.

24 Risner ME, etal: Efficacy of rosiglitazone in a genetically defined population with mild-to-moderate Alzheimer's disease. Pharmacogenomics J 2006;6:246-254.

25 Hanyu H, et al: Pioglitazone improved cognition in a pilot study on patients with Alzheimer's disease and mild cognitive impairment with diabetes mellitus. J Am Geriatr Soc 2009;57:177-179.

-26 Sato T, et al: Efficacy of PPAR-gamma agonist pioglitazone in mild Alzheimer disease. Neurobiol Aging 2011: 32:1626-1633.

27 Petersen RC: Mild cognitive impairment as a diagnostic entity. J Intern Med 2004;256:183-194.

-28 Executive Summary of the Third Report of the National Cholesterol Education Program (NCEP) Expert Panel on Detection, Evaluation, and Treatment of High Blood Cholesterol in Adults (Adult Treatment Panel III). JAMA 2001;285:2486-2497.

-29 Folstein MF, Folstein SE, McHugh PR: 'Mini-mental state'. A practical method for grading the cognitive state of patients for the clinician. J Psychiatr Res 1975;12:189-198.

30 Morris JC: The Clinical Dementia Rating (CDR): current version and scoring rules. Neurology 1993;43:24122414.

31 Galasko D, et al: An inventory to assess activities of daily living for clinical trials in Alzheimer's disease. The Alzheimer's Disease Cooperative Study. Alzheimer Dis Assoc Disord 1997;11(suppl 2):S33-S39.

-32 Radloff LS: The CES-D Scale: a self-report depression scale for research in the general population. Appl Psychol Meas 1977;1:385-401.

33 Wechsler D: WMS-R Wechsler Memory Scale - Revised Manual. New York, Harcourt Brace Jovanovich, 1987.

34 Rey RA: L'examen psychologique dans les cas d'encéphalopathie traumatique. Arch Psychol 1941;28:286340.

35 Kaplan EF: Boston Naming Test. Philadelphia, Lea \& Febiger, 1983.

36 Strauss E, Sherman EMS, Spreen 0: A Compendium of Neuropsychological Tests: Administration, Norms and Commentary, ed 3. New York, Oxford University Press, 2006

37 Wechsler D: Wechsler Adult Intelligence Scale - Revised. San Antonio, The Psychological Corporation, 1981.

38 Reitan RM: Validity of the Trail Making Test as an indicator of organic brain damage. Percept Mot Skills 1958; 8:271-276.

-39 Ivnik RJ, Malec JF, Smith GE, Tangalos EG, Petersen RC, Kokmen E, Kurland LT: Mayo's older Americans normative studies: WAIS-R norms for ages 56 to 97. Clin Neuropsychol 1992;6(suppl):1-30.

-40 Ivnik RJ, Malec JF, Smith GE, Tangalos EG, Petersen RC, Kokmen E, Kurland LT: Mayo's older Americans normative studies: WMS-R norms for ages 56 to 94. Clin Neuropsychol 1992;6(suppl):49-82.

41 Ivnik RJ, Malec JF, Smith GE, Tangalos EG, Petersen RC, Kokmen E, Kurland LT: Mayo's older Americans normative studies: updated AVLT norms for ages 56 to 97. Clin Neuropsychol 1992;6(suppl):83-104.

-42 Ivnik RJ, Malec JF, Smith GE, Tangalos EG, Petersen RC: Neuropsychological tests' norms above age 55: COWAT BNT, MAE Token, WRAT-R Reading, AMNART, STROOP, TMT and JLO. Clin Neuropsychol 1996;10:262-278.

-43 Malec JF, Ivnik RJ, Smith GE, Tangalos EG, Petersen RC, Kokmen E, Kurland LT: Mayo's older Americans normative studies: utility of corrections for age and education for the WAIS-R. Clin Neuropsychol 1992; 6(suppl):31-47.

44 Lucas JA, Ivnik RJ, Smith GE, Bohac DL, Tangalos EG, Graff-Radford NR, Petersen RC: Mayo's older Americans normative studies: category fluency norms. J Clin Exp Neuropsychol 1998;20:194-200.

$\$ 45$ Petersen RC, et al: Prevalence of mild cognitive impairment is higher in men. The Mayo Clinic Study of Aging. Neurology 2010;75:889-897.

46 Petersen RC, et al: Mild cognitive impairment due to Alzheimer disease in the community. Ann Neurol 2013; 74:199-208.

47 Mohs RC, et al: Development of cognitive instruments for use in clinical trials of antidementia drugs: additions to the Alzheimer's Disease Assessment Scale that broaden its scope. The Alzheimer's Disease Cooperative Study. Alzheimer Dis Assoc Disord 1997;11(suppl 2):S13-S21.

48 Van der Elst W, et al: The Stroop color-word test: influence of age, sex, and education; and normative data for a large sample across the adult age range. Assessment 2006;13:62-79.

49 Wechsler D: Wechsler Adult Intelligence Scale, ed 3. San Antonio, The Psychological Corporation, 1997. 
50 Esteban-Santillan C, et al: Clock drawing test in very mild Alzheimer's disease. J Am Geriatr Soc 1998;46: 1266-1269.

51 DeFronzo RA, Tobin JD, Andres R: Glucose clamp technique: a method for quantifying insulin secretion and resistance. Am J Physiol 1979;237:E214-E223.

-52 Van Pelt RE, et al: Acute modulation of adipose tissue lipolysis by intravenous estrogens. Obesity (Silver Spring) 2006;14:2163-2172.

53 Liu D, et al: Arterial, arterialized venous, venous and capillary blood glucose measurements in normal man during hyperinsulinaemic euglycaemia and hypoglycaemia. Diabetologia 1992;35:287-290.

54 Jiang Q, Heneka M, Landreth GE: The role of peroxisome proliferator-activated receptor-gamma (PPARgamma) in Alzheimer's disease: therapeutic implications. CNS Drugs 2008;22:1-14.

55 Gold M, et al: Rosiglitazone monotherapy in mild-to-moderate Alzheimer's disease: results from a randomized, double-blind, placebo-controlled phase III study. Dement Geriatr Cogn Disord 2010;30:131-146.

56 Harrington C, et al: Rosiglitazone does not improve cognition or global function when used as adjunctive therapy to AChE inhibitors in mild-to-moderate Alzheimer's disease: two phase 3 studies. Curr Alzheimer Res 2011;8:592-606.

57 Geldmacher DS, et al: A randomized pilot clinical trial of the safety of pioglitazone in treatment of patients with Alzheimer disease. Arch Neurol 2011;68:45-50.

58 Bergman RN, Finegood DT, Ader M: Assessment of insulin sensitivity in vivo. Endocr Rev 1985;6:45-86.

59 de la Monte SM, Wands JR: Alzheimer's disease is type 3 diabetes - evidence reviewed. J Diabetes Sci Technol 2008;2:1101-1113.

60 Shemesh E, et al: Effect of intranasal insulin on cognitive function: a systematic review. J Clin Endocrinol Metab 2012;97:366-376.

61 Rolland Y, Abellan van Kan G, Vellas B: Healthy brain aging: role of exercise and physical activity. Clin Geriatr Med 2010;26:75-87.

62 Angevaren M, et al: Physical activity and enhanced fitness to improve cognitive function in older people without known cognitive impairment. Cochrane Database Syst Rev 200;CD005381.

63 Lautenschlager NT, et al: Effect of physical activity on cognitive function in older adults at risk for Alzheimer disease: a randomized trial. JAMA 2008;300:1027-1037.

64 Baker LD, et al: Aerobic exercise improves cognition for older adults with glucose intolerance, a risk factor for Alzheimer's disease. J Alzheimers Dis 2010;22:569-579.

65 Cress ME, et al: Exercise: effects on physical functional performance in independent older adults. J Gerontol A Biol Sci Med Sci 1999;54:M242-M248.

66 Gozansky WS, et al: Protection of bone mass by estrogens and raloxifene during exercise-induced weight Loss. J Clin Endocrinol Metab 2005;90:52-59.

67 Kohrt WM, et al: Timing of ibuprofen use and bone mineral density adaptations to exercise training. J Bone Miner Res 2010;25:1415-1422.

68 Schenkman M, et al: Exercise for people in early- or mid-stage Parkinson disease: a 16-month randomized controlled trial. Phys Ther 2012;92:1395-1410.

69 Hildreth KL, et al: Effects of testosterone and progressive resistance exercise in healthy, highly functioning older men with low-normal testosterone levels. J Clin Endocrinol Metab 2013;98:1891-1900.

$\rightarrow 70$ Uemura K, et al: Cognitive function affects trainability for physical performance in exercise intervention among older adults with mild cognitive impairment. Clin Interv Aging 2013;8:97-102.

71 Bonneau GA, Pedrozo WR, Berg G: Adiponectin and waist circumference as predictors of insulin-resistance in women. Diabetes Metab Syndr 2014;8:3-7.

72 Zoico E, et al: Adipocytokines, fat distribution, and insulin resistance in elderly men and women. J Gerontol A Biol Sci Med Sci 2004;59:M935-M939.

73 Standards of medical care in diabetes - 2012. Diabetes Care 2012;35(suppl 1):S11-S63. 